\title{
Die Diffusion von Innovationen der Telematik in der Schweiz
}

\section{Einleitung}

Die Entwicklung und Verbreitung von technischem Fortschritt hat in verschiedensten Wissenschaftszweigen breite Beachtung gefunden. Die Notwendigkeit der Untersuchung von Diffusionsprozessen ergibt sich aus der Erkenntnis, daß solche Prozesse zu größeren Veränderungen der Verteilung der Wirtschaftsaktivitäten im Raum und der sozialen und politischen Struktur eines Landes führen können.

Die unterschiedliche Verbreitung von Innovationen wird deshalb als ein Erklärungsgrund für räumliche Disparitäten des Wohlstandes einer Volkswirtschaft angesehen (SCHMOOKLER 1972, NABSETH und RAY 1972). Die räumlich-zeitlich unterschiedliche Diffusion neuer Technologien kann wirtschaftliche Anpassungsreaktionen zur Folge haben, die bestimmend für die zukünftigen Entwicklungschancen einer Region sind. Diese strukturellen Veränderungen können wiederum Ursache für ein geringes Innovationspotential und die langsame Einführung neuer Technologien sein (MALECKı 1981), womit ein zirkulärer Prozeß beschrieben wäre.

Den Innovationen der Telematik, das sind Neuerungen in der Erfassung, Speicherung, Verarbeitung, Vervielfältigung, Übermittlung und Wiedergabe von Daten (also der Informationstechnologie und der Telekommunikation), kommt zweifellos eine große Bedeutung für die Entwicklung der Volkswirtschaft $\mathrm{zu}$, nimmt doch die Information in der nachindustriellen Gesellschaft eine zentrale Stellung ein (MACHLUP 1962).

\section{Ansätze der Diffusionsforschung}

Die Analyse der Diffusion von Neuerungen ist ein Forschungsthema, das von den meisten Sozialwissenschaften intensiv gepflegt wird. War es anfangs die Soziologie (TARDE 1895) und im speziellen die Agrarsoziologie, die den Großteil der Arbeiten beisteuerte, so hat 1953 Hägerstrand die Diffusionsforschung in eine neue Richtung gelenkt und viele weitere geographische Arbeiten induziert (HÄGERSTRAND 1953). Hägerstrand faßte die Adoption einer Innovation als das Ergebnis eines Lern- und Kommunikationsprozesses auf und simulierte dessen Ablauf mit verschiedenen Modellen. Die Betonung der
Bedeutung des Kommunikationsprozesses fand auch in anderen Forschungsansätzen Eingang, wie z. B. bei Lasuen, der die hierarchische Strukturierung des Informationsflusses im Raum hervorhob (LASUEN 1973) oder Pred, der auf die Bedeutung der wirtschaftlich-organisatorischen Strukturen in fortgeschrittenen Volkswirtschaften hinwies (PRED 1976).

Im Gegensatz dazu steht die ökonomische Diffusionsforschung, die die erwartete Profitabilität einer Innovation als zentralen Einflußfaktor betrachtet (GRILICHES 1957, MANSFIELD 1968). Zu einer Erweiterung der bisherigen Betrachtungsweise hat Brown beigetragen, indem er auf die Bedeutung der durch die adoptions- oder nachfrageorientierten Forschung vernachlässigten Restriktionen auf der Angebotsseite hinwies (BROWN 1981).

Wer die vielen empirischen Untersuchungen der erwähnten Forschungsrichtungen durchsieht, wird feststellen, daß zu den meisten gängigen Hypothesen über den Ablauf des Diffusionsprozesses widersprüchliche empirische Befunde vorliegen. Die Ursachen sind wohl darin zu suchen, da $\beta$ in den Modellen oft mit denselben Variablen verschiedene Hypothesen getestet werden (weil Indikatoren verwendet werden, die lediglich als Schätzgröße für die gesuchte Einflußvariablen dienen), und daß den spezifischen Eigenschaften einer Innovation und deren Ausbreitungsbedingungen zu wenig Aufmerksamkeit geschenkt wird. Ein weiterer Mangel scheint die fehlende Verknüpfung der Ansätze verschiedener Forschungsrichtungen zu sein (MÜDESPACHER 1984).

Während der Problematik des Auffindens geeigneter Indikatoren nicht völlig entronnen werden kann, soll in diesem Artikel ein Diffusionsmodell getestet werden, das die beiden letzten Kritikpunkte berücksichtigt.

\section{Die Innovationen der Telematik und ihre A usbreitungsbedingungen}

\subsection{Die neuen Technologien}

Die Innovationen der Informationstechnologie der letzten zwanzig Jahre sind durch die enormen Fort-

Alfred Müdespacher, Dr. oec. publ.,

ORL-Institut, ETH-Hönggerberg, 8093 Zürich 
schritte der Mikroelektronik, der Digitaltechnik und der Softwaretechnik ermöglicht worden. Diese Techniken werden heute sehr vielfältig eingesetzt. Wir konzentrieren uns in diesem Beitrag auf die Anwendung in der Datenverarbeitung und der Nachrichtenund Kommunikationstechnik.

Die Einführung der elektronischen Datenverarbeitung in den vierziger Jahren war wohl die markanteste Innovation der Informationstechnologie. Wurden anfangs vor allem Großanlagen eingesetzt, nehmen heute dank der erfolgreichen Miniaturisierung der elektronischen Schaltungen die sogenannten MikroComputer (dazu gehören Taschenrechner, Heimcomputer und Personal Computer) und die Mini-Computer (kleinere Anlagen, die z. B. als Prozeßrechner oder für die Büroadministration benutzt werden) zahlenmäßig auf dem Markt das Hauptgewicht ein. Dank den Neuerungen der Kommunikationstechnik kann die Leistung der EDV-Anlagen vervielfacht werden, indem die einzelnen Rechner miteinander verbunden werden. Solche Rechnerverbunde gibt es auf internationaler, nationaler und lokaler Ebene.

Die Neuerungen der Kommunikationstechnik umfassen die Verbesserungen des eigentlichen Netzes (der Kommunikationsinfrastruktur) und die Einführung neuer Dienstleistungen. Beim Netz wären die Umstellung von der Analog- auf die Digital-Technik, die Einführung des dienstintegrierten Netzes (ISDN) und die Glasfasertechnik (für Breitband-Übertragung) zu nennen. Ohne auf private Anbieter einzugehen, erwähnen wir von den neuen Diensten der schweizerischen PTT Telepac, Videotex, Teletex, automatische Meldungsvermittlung (SAM), Telefax (Fernkopierer) und die Datenanschlußmöglichkeit auf dem öffentlichen Wählnetz oder auf Mietleitungen'. Starke Zunahmen der Nachfrage werden für die nächsten 15 Jahre bei den Datenanschlüssen, Teletex und Telefax erwartet.

Wir untersuchen deshalb die Verbreitung des EDVEinsatzes der Tele-Datenfernverarbeitung und Telefax (Teletex eignet sich wegen der noch geringen Verbreitung nicht).

\subsection{Die Ausbreitungsbedingungen der Telematik}

Die untersuchten Neuerungen der Telematik wurden bisher fast ausschließlich kommerziell genutzt. Sie wurden als Prozeßinnovationen eingesetzt, waren also Verfahrensinnovationen in einem Produktionsprozeß. Die Prozeßinnovationen unterstehen anderen Diffusionsregeln als die Produktinnovationen, weil die potentiellen Adoptoren Unternehmen sind, die unter Konkurrenzdruck handeln und bei denen die Innovationsfähigkeit zur Existenzfrage werden kann. Während bei Produktinnovationen oft ein Einfluß des Standorts des Adoptors ausgemacht werden kann, spielen bei der Prozeßinnovation (vermutlich wegen des Konkurrenzdruckes) die regional unterschiedli- chen Adoptionsbedingungen eine geringere Rolle (EWERS 1984).

Wie MANSFIELD (1968) zeigte, stellt die erwartete Rentabilität bei Prozeßinnovationen eine wesentliche Einflußgröße für den Adoptionsentscheid dar. Ihre Schätzung gestaltet sich aber besonders bei komplexen Technologien, die strukturelle Anpassungen der Betriebsstruktur voraussetzen, äußerst schwierig, zumal die subjektive Erwartung und Bewertung zukünftiger technologischer Verbesserungen und Preisentwicklungen diese Größe stark beeinflußt.

Aus den Eigenschaften einer Innovation lassen sich weitere Hinweise über die Einflußfaktoren ihrer Diffusion ableiten. Die Neuerungen der Telematik setzen eine Normierung der Semantik voraus (APRILE et al. 1984). Deshalb eignen sich diese Neuerungen vor allem für den leicht standardisierbaren Teil der im kommerziellen Bereich zu verarbeitenden Information. Die Neuerungen werden folglich vor allem dort eingesetzt, wo standardisierte Informations- und Kommunikationsbeziehungen bereits bestehen oder leicht zu schaffen sind. Da Großbetriebe einen wesentlich höheren Kontroll-, Steuerungs- und Informationsbedarf und einen größeren Formalisierungsgrad der Entscheidungsabläufe aufweisen, werden sie die Neuerungen früher und schneller adoptieren. Dasselbe darf für multilokale Unternehmungen (Mehrbetriebsunternehmen) angenommen werden. Solche Betriebssysteme können als Transmissionsriemen des technischen Fortschritts in periphere Regionen wirken (PRED 1976).

Das gesamte Informationsvolumen einer Wirtschaftseinheit unterscheidet sich nun allerdings je nach ihrer Tätigkeit. Insbesondere variiert auch der Anteil an Routine-Informationstätigkeiten mit der Branchenzugehörigkeit. So weisen im zweiten Sektor die Metallund Maschinenindustrie, die chemische Industrie, die Uhren- und die Papierindustrie und im dritten Sektor die Banken, Versicherungen, die öffentliche Verwaltung und der Großhandel einen höheren Anteil an Routine-Informationstätigkeit auf (OECD 1981).

Das Angebot kann in zweifacher Hinsicht bei der Verbreitung der neuen Technologie eine Rolle spielen. Bei der Übermittlung von Daten ist die Abhängigkeit von der Telekommunikationsinfrastruktur leicht erkennbar. Eine gewichtige Einflußgröße dürfte hier die Tarifstruktur sein, falls distanzabhängige Tarife existieren. Die unterschiedliche Qualität und Kapazität des Netzes scheint für die untersuchten Technologien (noch) nicht von Bedeutung zu sein.

Die zweite Restriktion des Angebots ist durch die Verkaufsstellen gegeben, die die neue Technologie und die zugehörige Information vermitteln. Da es sich um eine komplexe Technologie handelt, dürfte die geographische Verteilung der sogenannten «Diffusion Agencies» nicht ohne Einfluß auf die Verbreitung der Innovationen sein (BROWN 1981). 
In der Verkaufsstelle kann sich ein potentieller Adoptor beraten lassen. Bessere Gewißheit über die Nützlichkeit der Innovation erhält er allerdings, wenn er sie im praktischen Einsatz funktionieren sieht. Wir betrachten deshalb die Lokalisationsvorteile, ausgedrückt als Anteil der Betriebe einer Branche in einer Region, wegen des möglichen Informationsaustausches als adoptionsfördernd.

Das Netz der ökonomischen Aktivitäten weist meist ein räumlich hierarchisches Gefüge auf und steht nach Lasuen direkt mit dem Fluß der innovationsbezogenen Informationen in Beziehung (LASUEN 1973). Diese Hierarchie müßte denn auch im Diffusionsmuster zu erkennen sein. Für die Informationstechnologie gilt in besonderem $\mathrm{Maße}$, daß ihre Verbreitung zuerst in den Zentren und ihrem Umland und erst später in den peripheren Regionen erwartet wird. Diese Technologie wird nämlich in den Zentren entwickelt und zuerst von dort aus vertrieben. Außerdem sind nur in den Zentren genügend Fachkräfte zu finden und liegt der Informations- und Kommunikationsbedarf in den peripheren Regionen tiefer (APRILE et al. 1984).

\section{Die Verbreitung der Innovationen der Informations- technologie in den Betrieben}

\subsection{Die Daten}

Die verwendeten Daten für die EDV-Untersuchung entstammen einer Umfrage des Institutes für Automation und Operations Research der Universität Freiburg und des Institutes für Marktforschung in Hergiswil bei 10743 schweizerischen Betrieben (Rücklaufquote von $51,4 \%=5518$ Betriebe $)^{2}$. Da eine stratifizierte Zufallsstichprobe aufgrund der Betriebszählung 1975 (ohne Landwirtschaft) gezogen wurde, haben wir die eingegangenen Fragebogen je MS-Region ${ }^{3}$ nach Betriebsgrößenklasse, Wirtschaftssektor und Raumtyp gewichtet. Die Angaben über die Nutzung von EDV in den Betrieben beziehen sich auf das Stichdatum 31.12.1983. Der gesamte Kanton Tessin und die Region Mesolcina ist wegen den hohen Übersetzungskosten nicht befragt worden. Die Daten für die Untersuchung der Diffusion der Telefax-Geräte sind den entsprechenden Verzeichnissen entnommen wor$\operatorname{den}^{4}$.

\subsection{Der Einsatz von EDV in den Regionen der Schweiz}

In der EDV-Umfrage wurde danach gefragt, ob im Betrieb EDV in irgendeiner Form genutzt wird, sei das mit einem eigenen Computer im Hause oder mittels Fernverarbeitung. Die Abbildung 1 zeigt die

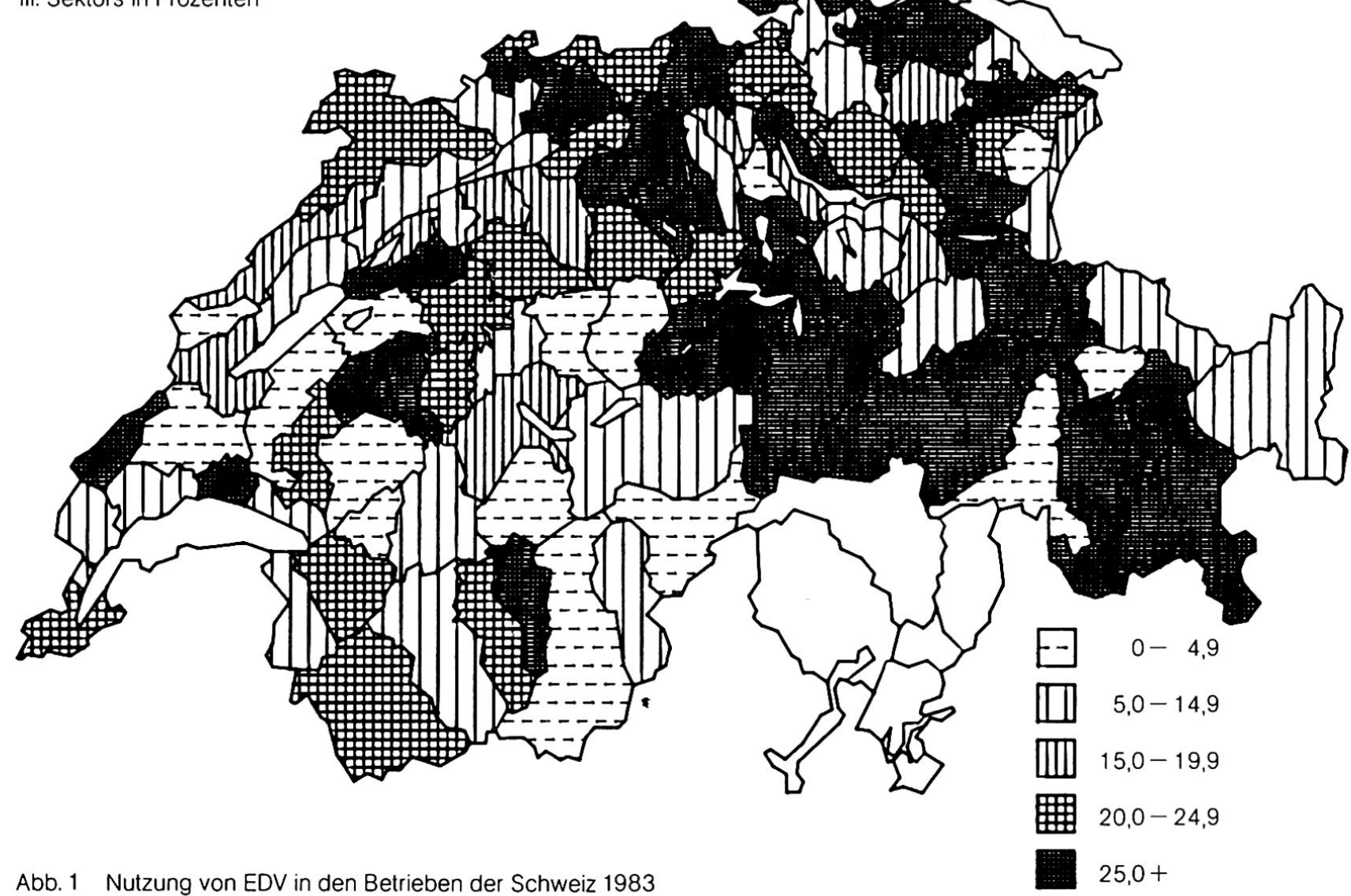


Anteile der Betriebe mit EDV-Einsatz am Total der Betriebe (ohne Landwirtschaft) auf. Die Regionen mit überdurchschnittlichem EDV-Einsatz gehören zu den Großzentren, den zentrennahen und den touristischen Gebieten. Wir finden darunter aber auch tertiäre Kleinzentren (Uri, Nidwalden-Engelberg) und sogar Gebiete, die eher zur agrarisch-industriellen und -touristischen Peripherie gehören (Erlach-Seeland, Toggenburg, Surselva). Wegen der geringen Zahl der befragten Betriebe wurde die Hochrechnung in diesen Regionen zum Teil ungenau. Wäre der Landwirtschaftssektor in die Untersuchung einbezogen worden, würden einige dieser Gebiete eine wesentlich geringere Dichte aufweisen.

Faßt man die Regionen nach 12 Raumtypen zusammen (SCHULER und NEF 1983), so zeigt sich, daß rund $31 \%$ aller Betriebe mit EDV-Einsatz ihren Standort in den fünf Großzentren haben. In den Großzentren, ihrem Umfeld und den Mittelzentren liegen rund $64 \%$ der Betriebe mit EDV-Einsatz.

Die Betriebe mit Computer im Hause sind stärker konzentriert als die Betriebe mit EDV-Einsatz. Die Großzentren, ihr Wohn- und Arbeitsplatz-Umland und die tertiären Mittelzentren weisen einen deutlich überdurchschnittlichen Anteil dieser Betriebe auf (insgesamt die Hälfte aller Betriebe mit einem Computer liegt in diesen Gebieten).

Die Verteilung der Betriebe mit Personal Computers fällt noch einseitiger aus. Rund $43 \%$ aller Betriebe mit solchen Geräten befinden sich in den fünf Großstadtzentren.

\subsection{Die Nutzung der Tele-Datenfernverarbeitung}

Die Abbildung 2 zeigt die relative Verteilung der Betriebe, die mittels Telekommunikation Daten fernverarbeiten. Die Nutzung der Tele-Fernverarbeitung konzentriert sich räumlich stärker auf die großen und mittleren Zentren als etwa die Verteilung der Betriebe mit Computern und insbesondere der Betriebe mit EDV-Einsatz. Ausnahmen sind auch hier wieder festzustellen (Uri, Surselva usw.), allerdings fallen diese Regionen in absoluten Zahlen nicht ins Gewicht. Insgesamt haben rund $70 \%$ der Betriebe mit Tele-Fernverarbeitung ihren Standort in den Großzentren, ihrem Umland oder den Mittelzentren.

Anteil der Betriebe mit Tele-Fernverarbeitung am Total der Betriebe des II. + III. Sektors in Prozenten

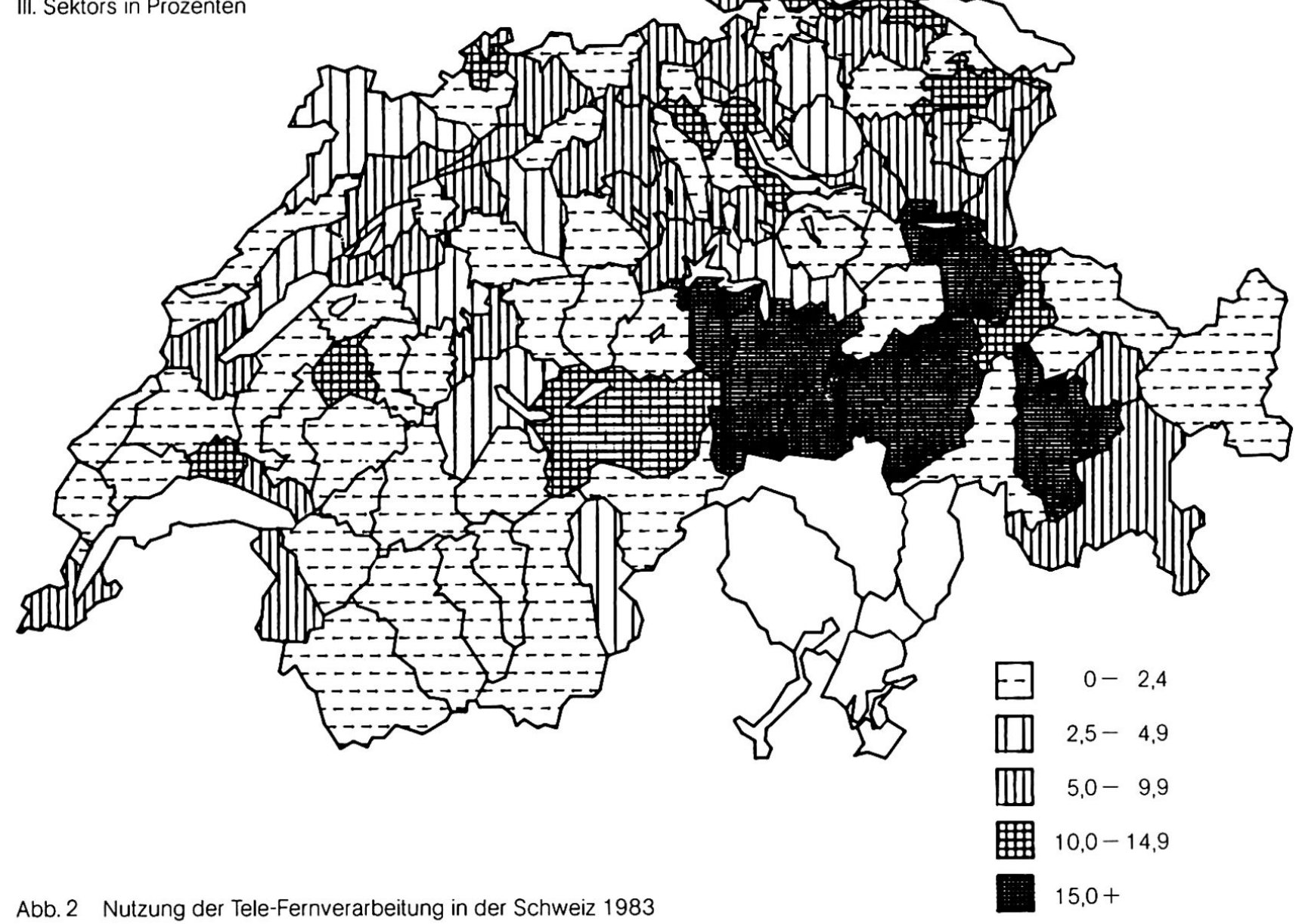




\subsection{Der Einsatz von Fernkopierern}

Fernkopierer sind Telekommunikations-Endgeräte, die auf einzelnen Seiten enthaltene Informationen übermitteln und sie beim Empfänger wieder in derselben Form reproduzieren. Sie benutzen dazu den Telefax-Dienst der PTT, der am 14.7.1980 versuchsweise eingeführt worden ist (definitiver Betrieb ab 1.1.1983).

In der erfaßten Zeitperiode (1980-84) wurden drei verschiedene Geräteklassen von Fernkopierern eingesetzt; die Geräte der Gruppen 1 bis 3. Die Geräte der Gruppe 1 sind nicht nach internationaler Norm (CCITT) standardisiert und deshalb mit den anderen Geräten nicht kompatibel. Sie sind im PTT-Verzeichnis nicht eingetragen und wurden von den PTT nicht vermietet. Ihre Übertragungsdauer beträgt 6 Minuten pro A4-Seite. Heute sind nur noch wenige Geräte in Betrieb. Die Übertragungsdauer der Gruppe-2-Geräte beträgt 3 Minuten je A4-Seite. Sie können mit den Geräten der Gruppe 3 kommunizieren. Die Geräte der Gruppe 3 nützen die maximal mögliche Übertragungsrate des Telefon-Wählnetzes von $9600 \mathrm{Bit} / \mathrm{s}$ aus. Sie verfügen über ein «intelligentes» Modem, das die Übertragungsgeschwindigkeit automatisch der Leitungsqualität anpaßt. Sie benötigen mindestens 30 Sekunden für eine A4-Seite. Die Geräte sind seit der zweiten Hälfte 1983 im Fachhandel erhältlich. Die PTT vermieten sie seit 1984 . Sämtliche Geräte (auch die privat gekauften) müssen den PTT gemeldet werden. Die Zahl der angeschlossenen Geräte ist aus Tabelle 1 ersichtlich.

Tabelle 1 Telefax-Geräte 31.10.1984

\begin{tabular}{|l|lr|}
\hline PTT-Geräte & Gruppe 2 & 547 \\
\cline { 2 - 3 } & Gruppe 3 & 460 \\
\hline Private Geräte & Gruppe 1 & 228 \\
\cline { 2 - 3 } & Gruppe 2 & 411 \\
\cline { 2 - 3 } & Gruppe 3 & 833 \\
\hline Total & & 2479 \\
\hline
\end{tabular}

Quelle: PTT

In Abbildung 3 sind die Adoptionsraten der Fernkopierer (Gruppe 2 und 3) dargestellt. Wiederum zeigt sich eine deutliche Konzentration auf die Großzentren und ihr Arbeitsplatz-Umland sowie die Mittelzentren. Zu Beginn des Diffusionsprozesses (1980) stand mit einer Ausnahme (oberes Emmental) in keiner peripheren Region ${ }^{5}$ ein Telefax-Gerät.

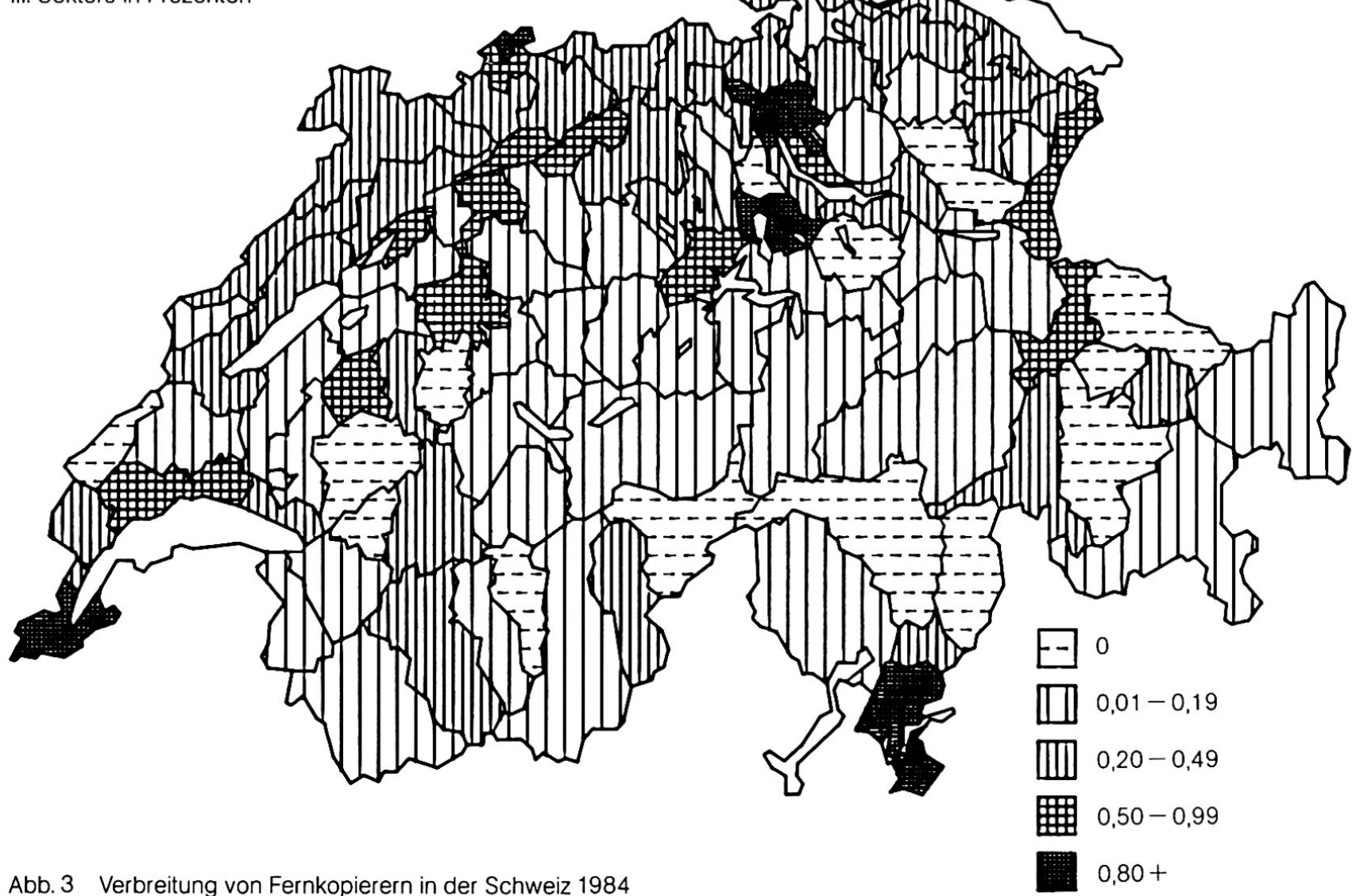


5. Die Ursachen unterschiedlicher räumlicher Diffusion von Innovationen der Informationstechnologie

\subsection{Die Diffusion der elektronischen Daten- verarbeitung in Betrieben}

Die eingangs erwähnten Einflußgrößen der Diffusion werden mit einem Schätzmodell auf ihren tatsächlichen Einfluß getestet. Wir stellen für die beiden untersuchten Wirtschaftssektoren zwei verschiedene Schätzgleichungen auf, da die Bedingungen für den Einsatz von EDV im zweiten und dritten Sektor recht unterschiedlich sind (z. B. Anteil an Routine-Informationstätigkeiten) und außerdem einige Daten nur für die Industrie verfügbar waren.

Die Schätzgleichung für den Test mit einer Diskriminanzanalyse lautet für die Industrie:

$$
\begin{aligned}
\mathrm{D}_{\mathrm{I}}= & \mathrm{b}_{\mathrm{o}}+\mathrm{a}_{1} \cdot \text { BESCH }+\mathrm{a}_{2} \cdot \text { TELPREIS }+\mathrm{a}_{3} \cdot \text { EINK } \\
& +\mathrm{a}_{4} \cdot \text { LOK }+\mathrm{a}_{5} \cdot \text { ROUT }+\mathrm{a}_{6} \cdot \text { DBANK }+\mathrm{a}_{7} \cdot \\
& \text { SERV }+\mathrm{a}_{8} \cdot \text { HARD }+\mathrm{a}_{9} \cdot \text { BNPI81 }+\mathrm{a}_{10} \\
& \text { AHAUPI81 }+\mathrm{a}_{11} \cdot \text { ADEPI81 }+\mathrm{A}_{12} \cdot \mathrm{ZEN} \\
& \text { DUM } 1+\mathrm{a}_{13} \cdot \text { ZENDUM }+\mathrm{a}_{14} \cdot \text { ZENDUM3 } \\
& +\mathrm{a}_{15} \cdot \text { WIRTINT }+\mathrm{u}
\end{aligned}
$$

\begin{tabular}{|c|c|}
\hline D & - Diskriminanzwert \\
\hline $\mathrm{BESCH}$ & $=$ Anzahl der Beschäftigten des Betriebes \\
\hline TELPREIS & $\begin{array}{l}\text { Durchschnittlicher Preis für ein Telefonge- } \\
\text { spräch von einer Minute Dauer aus der } \\
\text { Netzgruppe des Betriebs zu den nächsten } \\
\text { drei Großzentren; das nächstgelegene } \\
\text { Großzentrum ist doppelt gewichtet. }\end{array}$ \\
\hline EINK & $\begin{array}{l}\text { - Persönlich verfügbares Einkommen je Ein- } \\
\text { wohner } 1980 \text { in Franken (Fischer 1984). } \\
\text { Indikator für ökonomische Hierarchie. }\end{array}$ \\
\hline LOK & $\begin{aligned} &= \text { Anteil der Industrie- oder Dienstleistungs- } \\
& \text { betriebe am Total der Betriebe der Region } \\
& \text { (= Lokalisationsvorteil). }\end{aligned}$ \\
\hline ROUT & $\begin{array}{l}\text { - Dummy-Variable für die Zugehörigkeit des } \\
\text { Betriebs zu einer informationsintensiven } \\
\text { Branche (in } 3.2 \text { definiert). }\end{array}$ \\
\hline WIRTINT & $\begin{array}{l}=\text { Anzahl Arbeitsplätze (1975) dividiert durch } \\
\text { die Einwohnerzahl (1980) = Wirtschaftsin- } \\
\text { tensität; Indikator für ökonomische Hierar- } \\
\text { chie. }\end{array}$ \\
\hline DBANK & $\begin{aligned} \text { - Anteil der Bankbetriebe am Total der } \\
\text { Betriebe des zweiten und dritten Sektors in } \\
\text { der Region (steht für die Finanzierungs- } \\
\text { möglichkeiten). }\end{aligned}$ \\
\hline SERV & $\begin{array}{l}\text { - Gibt an, ob mindestens ein EDV-Beratungs- } \\
\text { oder Softwarebetrieb in der Region ansäs- } \\
\text { sig ist. }\end{array}$ \\
\hline
\end{tabular}

Die Schätzgleichung für den Dienstleistungssektor hat folgende Form:

$$
\begin{aligned}
\mathrm{D}_{\mathrm{DL}}= & \mathrm{b}_{0}+\mathrm{a}_{1} \cdot \text { BESCH }+\mathrm{a}_{2} \cdot \text { TELPREIS }+\mathrm{a}_{3} \cdot \\
& \text { EINK }+\mathrm{a}_{4} \cdot \text { LOK }+\mathrm{a}_{5} \cdot \text { ROUT }+\mathrm{a}_{6} \cdot \text { HARD } \\
& +\mathrm{a}_{7} \cdot \text { BUEROSOFT }+\mathrm{a}_{8} \cdot \text { ZENDUM } 1+\mathrm{a}_{9} \cdot \\
& \text { ZENDUM2 }+\mathrm{a}_{10} \cdot \text { ZENDUM }+\mathrm{a}_{11} \cdot \\
& \text { WIRTINT }+\mathrm{u}
\end{aligned}
$$

Die Variablen sind wie folgt definiert:

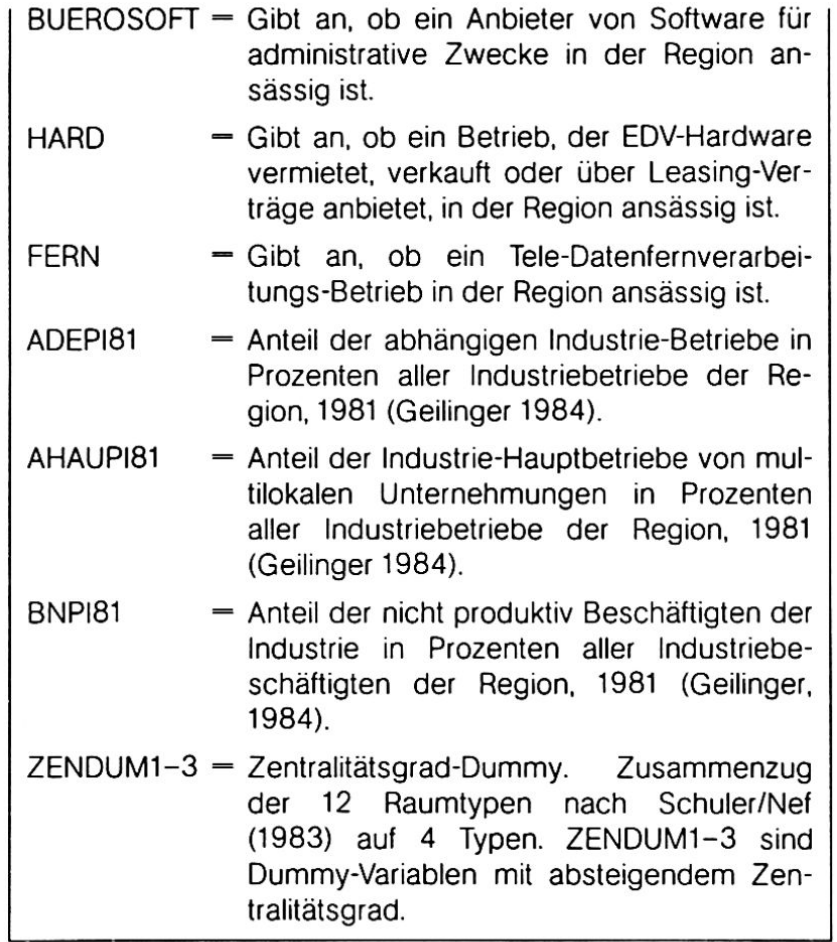

Bei beiden Schätzungen (vgl. Tabelle 2) kann aufgrund des F-Tests der Lageunterschiede der beiden Gruppen praktisch mit Sicherheit ausgesagt werden, dass die Diskriminanzfunktionen echten Lageunter-

Tabelle 2 Diskriminanzanalysen des zweiten und dritten Sektors: Betriebe ohne und mit EDV-Einsatz (Standardisierte Funktion)

\begin{tabular}{|l|l|l|}
\hline Variablen & $\begin{array}{l}\text { Sektor } 2 \\
\text { Koeffizienten }\end{array}$ & $\begin{array}{l}\text { Sektor } 3 \\
\text { Koeffizienten }\end{array}$ \\
\hline BESCH & $0.858^{\star \star}$ & $0.422^{\star \star}$ \\
TELPREIS & $-0.259^{\star}$ & $0.178^{\star \star}$ \\
EINK & $0.330^{\star \star}$ & - \\
ROUT & - & $0.893^{\star \star}$ \\
DBANK & $0.212^{\star}$ & - \\
HARD & $-0.193^{\star}$ & -0.134 \\
BNPI81 & $-0.268^{\star}$ & n. a. \\
AHAUPI81 & 0.176 & n. a. \\
FERN & - & $0.209^{\star \star}$ \\
BUEROSOFT & n.a. & $0.199^{\star}$ \\
ZENDUM1 & $0.422^{\star \star}$ & - \\
ZENDUM2 & $0.471^{\star \star}$ & - \\
\hline Signifikanz der & & \\
Gruppenunter- & 0.0000 & 0.0000 \\
schiede & & \\
\hline Wilk's Lambda/ & 0.9121 & 0.8571 \\
Signifikanz & 0.0000 & 0.0000 \\
\hline Richtige & $74,5 \%$ & $65,8 \%$ \\
Klassierung & & 0.15 \\
\hline$R^{2}$ & 0.09 & \\
\hline
\end{tabular}

** Signifikant mit weniger als $1 \%$ Irrtumswahrscheinlichkeit

* Signifikant mit weniger als $5 \%$ Irrtumswahrscheinlichkeit Ohne Stern: Signifikant mit weniger als 10\% Irrtumswahrscheinlichkeit 
schieden der beiden Gruppen entsprechen. Der ChiQuadrat-Test des Wilk's Lambda belegt mit der hohen Signifikanz die Bedeutung der gewählten Variablen. Wilk's Lambda ist ein inverses Mass der Trennkraft der Variablen. $\mathrm{Da}$ es in beiden Schätzungen nahe bei 1 liegt, können wir daraus schliessen, dass neben den gewählten Variablen weitere wichtige Einflussgrößen existieren. Darauf weist auch das tiefe Bestimmtheitsmass $\left(\mathrm{R}^{2}\right)$ hin, das den Anteil der durch die Gruppenzugehörigkeit erklärten Varianz der Variablen angibt. Die in den Schätzungen fehlenden wichtigen Einflussgrössen vermuten wir in jenen Eigenheiten der Betriebe und der Innovation, die wir mangels Angaben nicht ins Modell einbauen konnten. Solche Einflussgrössen wären etwa die erwartete Rentabilität, die Investitionssumme (MANSFIELD 1968), Finanzierungsverhältnisse (NABSETH UND RAY 1972), die Ertragslage des Betriebs, der Konkurrenzdruck (KENNEDY und THIRLWALL 1972), Abhängigkeiten und Vernetzungen der Betriebe oder die Risikobereitschaft des Unternehmers.

Gleichwohl ist die Bedeutung der Funktionen durch ihre Signifikanz gegeben. Die Koeffizienten der Variablen und ihr partieller F-Wert werden deshalb nachfolgend interpretiert:

Industriesektor: Die einflußreichste Variable ist die Anzahl der Beschäftigten. Sie weist neben den größten Koeffizienten (der in der standardisierten Funktion das relative Gewicht der Variable angibt) auch den deutlich höchsten partiellen F-Wert auf. Danach folgen im Einfluß bereits die beiden räumlichen Dummies, die den innovationsfördernden Einfluß eines Standorts in den nicht peripheren Gebieten belegen. Auch das persönlich verfügbare Einkommen weist auf die räumlich-hierarchische Komponente des Diffusionsprozesses hin.

Etwas weniger signifikant sind der Bankenanteil, die PTT-Taxen, der Anteil des unproduktiven Personals und das Vorhandensein einer Verkaufsstelle für Hardware. Alle Variablen haben etwa ähnlichen Einfluß, die beiden letzteren aber entgegen unseren Erwartungen ein negatives Vorzeichen. HARD muss als Korrekturfaktor zu den beiden räumlichen Dummies verstanden werden, denn sein partieller F-Wert steigt erst mit Einbezug dieser Variablen an. BNPI81 ist eine Korrekturgröße zu EINK, was so interpretiert werden kann, daß arme Regionen ein (noch) großes Rationalisierungspotential für EDV (angezeigt durch hohes BNPI81) aufweisen. Reichere Regionen haben hingegen ein höheres BNPI81, weil dort die Führungsfunktionen zentralisiert sind (GEILINGER 1984). Der Einfluß des Anteils der Hauptbetriebe ist unsicher. Tendenziell scheinen aber doch die Innovationen zuerst beim Mutterbetrieb stattzufinden.

Für die Gestaltung der Fernmeldepolitik ist von Bedeutung, daß die distanzabhängigen Gesprächstaxen auf dem Wählnetz einen signifikant negativen Einfluß auf den Adoptionsentscheid ausüben.
Dienstleistungssektor: Im Gegensatz zum zweiten Sektor ist bei den Dienstleistungen die Zugehörigkeit zu einer informationsintensiven Branche der bedeutendste und sehr hoch signifikante Einflußfaktor. Dies kommt nicht überraschend, differiert doch der Anteil an Routine-Informationstätigkeiten in der Industrie im Unterschied zum sehr heterogenen Dienstleistungssektor viel weniger stark zwischen den einzelnen Branchen. Dafür hat die Betriebsgröße geringeres Gewicht - je informationsintensiver die Branche, desto eher lohnt sich die EDV-Einführung auch für mittlere oder sogar kleinere Betriebe. Diese beiden Variablen sind mit Abstand die bedeutendsten.

FERN hat das erwartete Vorzeichen, TELPREIS hingegen nicht. Aufgrund des Verhaltens des partiellen F-Wertes von TELPREIS kann dies nur so interpretiert werden: Die Existenz eines Tele-Datenverarbeitungsbetriebes hat eindeutig einen positiven Einfluß auf den Adoptionsentscheid. Dies kommt bei der Industrie nicht zum Ausdruck, weil die TeleFernverarbeitung hauptsächlich für kleinere bis mittlere Betriebe Bedeutung hat. Besonders positiv ist die Existenz eines Fernverarbeitungsbetriebes, wenn es sich um eine Region mit einem hohen Übermittlungspreis, also um Peripherie handelt|(TELPREIS wird nur zusammen mit FERN signifikant).

HARD hat wiederum ein negatives Vorzeichen, der Einfluß ist aber weniger signifikant. Es handelt sich eindeutig um einen Korrekturfaktor von BUEROSOFT. Offenbar geht kein positiver Einfluß von einer Verkaufsstelle für Hardware aus. Die Geräte werden wohl oft direkt vom Importeur oder Hersteller bezogen. Hingegen scheint die Existenz einer Stelle für Bürosoftware und die dazugehörige Beratung ein gewisses Gewicht einzunehmen.

\subsection{Die Diffusion der Nutzung der Tele-Datenfern- verarbeitung in Betrieben}

In einer separaten Schätzung beschäftigen wir uns mit einer speziellen Form der Datenverarbeitung, die nicht nur die Technologie der Informationsverarbeitung, sondern ebenso die Neuerungen der Telekommunikation nutzt. Die Verbreitung der Fernverarbeitung mittels Telekommunikation unterliegt prinzipiell denselben Einflußfaktoren wie die Diffusion des Einsatzes von EDV. Wir verwenden deshalb die im Dienstleistungssektor eingesetzte Funktion und ersetzen einzig die Variable BUEROSOFT durch SERV. Wir erwarten einen stärkeren Einfluß der Fernverarbeitungsbetriebe und der PTT-Taxen. Außerdem dürfte der Einfluß der Betriebsgröße sinken.

Die Schätzung zeigt in etwa das erwartete Bild. Die Betriebsgröße hat einen kleineren Koeffizienten als in den beiden vorangegangenen Berechnungen. FERN hingegen erhielt ein höheres Gewicht. Standorte in peripheren Regionen beeinflussen die Adoption negativ. Die periphere Lage wirkt sich also - wohl aus 
verschiedenen Gründen (teurere PTT-Taxen, fehlende Information, Risikobereitschaft, fehlende Kontakte mit Lieferanten usw.) hemmend aus. Ist jedoch ein Fernverarbeitungsbetrieb in der Region vorhanden, so kann der negative Einfluß - zwar nicht ganz vollständig - korrigiert werden, da in diesem Fall einige Argumente wegfallen. Die PTT-Taxen treten in dieser Schätzung nur indirekt (über FERN und ZENDUM3) in Erscheinung. Den wichtigsten Einfluß übt jedoch die Zugehörigkeit zu einer Branche mit hohem Anteil an Routine-Informationstätigkeiten aus. Interessant ist außerdem, daß die Lokalisationsvorteile (mit mäßigem positivem Gewicht) signifikant werden. Vielleicht hängt dies mit der eher kleineren Betriebsgröße der Adoptoren zusammen, womit sich die Informationskanäle eher auf die regionale Ebene beschränken. Die Wirtschaftsintensität vertritt in dieser Schätzung mit mittlerem Gewicht die hierarchische Komponente des Diffusionsprozesses. Alle erwähnten Variablen sind mit weniger als $1 \%$ Irrtumswahrscheinlichkeit signifikant. Die Trefferrate beträgt rund $68 \%$.

\subsection{Die Diffusion von Fernkopierern}

Die Verbreitung der Fernkopierer wurde von 1980-1984 erhoben. Die beobachtete Größe war die Anzahl der Betriebe je MS-Region, die über mindestens ein Gerät verfügen. Diese Datenreihe erlaubt damit Aussagen über den Diffusionsprozeß.

Mit dem Kolmogoroff-Smirnoff-Test kann überprüft werden, ob die Adoptionsrate der Fernkopierer (Betriebe mit Fernkopierer dividiert duch das Total der Betriebe des zweiten und dritten Sektors) gleichmässig über alle Regionen verteilt ist oder ob sich räumliche Ungleichheiten zeigen. Für alle fünf Jahre sagt der Test aus, daß mit einer Irrtumswahrscheinlichkeit von weniger als 0.0001 solche Ungleichheiten existieren, wie wir sie bereits in 4.4 darstellten. Interessant ist jedoch, daß diese ungleiche Verbreitung sich mit der Dauer des Innovationsprozesses abschwächt. Ein geeignetes Maß für die Konzentration ist der Gini-Index.

Aus Tabelle 3 ist deutlich ersichtlich, daß die räumliche Konzentration zu Beginn des Diffusionsprozesses höher lag und sich dann rasch verminderte. Zwischen 1982 und 1984 fand jedoch nur noch eine leichte Dekonzentration statt. (Beim Gini-Index be-

Tabelle 3 Gini-Index: Räumliche Konzentration der Betriebe mit Fernkopierern (MS-Regionen)

\begin{tabular}{|l|l|}
\hline Jahr & Gini-Index \\
\hline 1980 & 0.833 \\
1981 & 0.783 \\
1982 & 0.748 \\
1983 & 0.745 \\
1984 & 0.738 \\
\hline
\end{tabular}

deutet Wert von 1 die Konzentration aller Geräte auf eine Region und der Wert von 0 eine uniforme Verteilung.) Diese Abnahme der Konzentration der Innovationsverbreitung wurde auch in Untersuchungen über andere Diffusionsprozesse bestätigt (vgl. z. B. EWERS 1984).

Mit einer weiteren Diskriminanzanalyse gingen wir den Ursachen der fehlenden Adoption in gewissen Regionen nach. Die 106 Regionen wurden zu diesem Zweck in zwei Gruppen geteilt: Jene, in denen bereits Betriebe Fernkopierer anwenden, und jene, in denen dies (noch) nicht der Fall ist.

Folgende Diskriminanzfunktion wurde getestet:

$\mathrm{D}=\mathrm{b}_{0}+\mathrm{a}_{1} \cdot$ GRUG $+\mathrm{a}_{2} \cdot$ AROUT $+\mathrm{A}_{3} \cdot$ WIRTINT $+a_{4} \cdot$ HIER $+a_{5} \cdot$ HARD $+a_{6}$.

AHAUPI81 $+a_{7} \cdot$ EINK $+a_{8} \cdot$ ZENDUM1 $+a_{9}$ ZENDUM2 $+\mathrm{a}_{10} \cdot$ ZENDUM $3+\mathrm{a}_{11} \cdot$ TELPREIS + u

GRUG = Anteil der Betriebe mit über 100 Beschäftigten;

AROUT = Anteil der Betriebe mit viel RoutineInformationstätigkeit;

TELPREIS = Übermittlungspreis für 1 A4-Seite; alles übrige wie bisher definiert.

Das Schätzergebnis für das erste Jahr (1980) ist in Tabelle 4 dargestellt. Die Funktion ist hoch signifikant, die Variablen zeigen eine gute Trennkraft, es wird immerhin etwa $50 \%$ der Varianz der Variablen durch die Gruppenzugehörigkeit erklärt, und die Trefferrate beträgt $84 \%$.

Die Variablen tragen alle ein ähnliches Gewicht. Die Vorzeichen entsprechen den Erwartungen. Als sehr gut abgesichert erweisen sich die räumlichen Variablen und die Variable für die ökonomisch-hierarchische Struktur des Diffusionsprozesses (WIRTINT).

Tabelle 4 Diskriminanzanalyse: Regionen mit und ohne Betriebe mit Fernkopierern 1980

\begin{tabular}{|l|c|}
\hline Variable & Koeffizienten \\
\hline AROUT & $0.433^{\star}$ \\
WIRTINT & $0.377^{\star \star}$ \\
TELPREIS & $-0.361^{\star}$ \\
ZENDUM1 & $0.452^{\star \star}$ \\
ZENDUM3 & $-0.401^{\star \star}$ \\
\hline Signifikanz der & 0.0000 \\
Gruppenunterschiede & $\begin{array}{l}0.520 / \\
\text { Wilk's Lambda/ }\end{array}$ \\
Signifikanz & 0.0000 \\
\hline Richtige & $84 \%$ \\
\hline Klassierung & 0.48 \\
\hline$R^{2}$ & \\
\hline
\end{tabular}

** Signifikant mit weniger als $1 \%$ Irrtumswahrscheinlichkeit * Signifikant mit weniger als 5\% Irrtumswahrscheinlichkeit 
$\mathrm{Zu}$ Beginn der Verbreitung zeichnet sich also die räumliche Struktur recht deutlich ab: die mittelzentralen Regionen bieten ein innovationsförderndes Umfeld, während von den hochzentralen und den kleinzentralen Gebieten keine besonderen Impulse ausgehen und die peripheren Standorte hemmend auf die Adoptionsbereitschaft wirken.

Etwas weniger signifikant zeigt sich der Anteil von Branchen mit viel Routine-Informationstätigkeiten: Durchaus verständlich, wenn man bedenkt, daß die Telefax-Geräte minimale Anforderungen an die Standardisierbarkeit der Information stellen.

Auch bei Telefax erweist sich der distanzabhängige Preis für die Übertragung als hemmend auf die räumliche Ausbreitung der Innovation. Den weiteren Variablen wie EINK, AHAUPI81 und HARD kommt keine Bedeutung zu. Die erste Variable würde einen Einfluß wohl eher bei Produktinnovationen geltend machen (bei der das Nachfragepotential der Bevölkerung eine Rolle spielt), die Industrievariable AHAUPI81 kann in einer Gesamtschätzung nicht durchdringen, und ein Einfluß von HARD wird wegen der Mietmöglichkeit bei den PTT verhindert. Bezieht man jedoch nur die nicht bei den PTT gemieteten Geräte in die Analyse ein, so zeigt sich ein signifikanter Einfluß von HARD (jedoch nur mäßiges Gewicht). Interessant ist, daß ein hoher Anteil an großen Unternehmungen mindestens zu Beginn der Verbreitung keine Rolle spielt.

Wie die vorangegangenen Berechnungen zeigen, verändern sich offensichtlich im Laufe des Diffusionsprozesses die räumlichen Einflußfaktoren. Schätzungen, die für die Jahre 1981-1984 durchgeführt wurden, bestätigen, daß der negative Einfluß der peripheren Standorte im Laufe der Zeit abnimmt. Das Gewicht des Anteils der Betriebe mit viel RoutineInformationstätigkeiten erhöht sich. Der Übertragungspreis verliert an Signifikanz, sobald die schnelleren Geräte der Gruppe 3 eingeführt werden. Hingegen erhält der Anteil der Großbetriebe einen signifikanten und bedeutenden Einfluß. Betrachtet man die Situation 1984 , so zeigt sich, daß sich nur noch einige kleinzentrale und periphere Regionen, in denen kaum größere Betriebe oder Betriebe der informationsintensiven Branchen angesiedelt sind, der Adoption der Innovation Telefax ganz entziehen können.

\section{Regionalwirtschaftliche Bedeutung der Ergebnisse}

Die Resultate der Analyse haben zu einigen der vermuteten Wirkungszusammenhänge empirische Evidenz geschaffen.

Als durchwegs bedeutsam für den Diffusionsprozeß in der Informationstechnologie hat sich die Betriebsgröße gezeigt: Größere Betriebe innovieren häufiger. Unklar bleibt hingegen nach wie vor der dahinter stehende ursächliche Zusammenhang: Sind größere Betriebe risikofreudiger, haben sie ein dickeres
Finanzpolster, informieren sie sich besser, oder liegt der Grund an ihren größeren Einsatzmöglichkeiten für die Informationstechnologie? Der Einfluß der Betriebsgröße schwächt sich mit zunehmendem Alter des Diffusionsprozesses ab.

Die Bedeutung des Anteils an Routine-Informationstätigkeiten und damit der Branchenzugehörigkeit hat sich erwartungsgemäß ebenso bestätigt. Während im zweiten Sektor ein deutlicher Effekt über den Raumtypus oder die Variablen eines ökonomisch-hierarchischen Prozesses feststellbar war, konnte dies für den Dienstleistungssektor nicht nachgewiesen werden. Bei beiden Sektoren zeigte sich jedoch, daß distanzabhängige Telekommunikationsgebühren die Entwicklung in der Peripherie hemmen, es sei denn, es entstünden auch in den peripheren Räumen Rechenzentren für die Tele-Datenfernverarbeitung.

Die Bedeutung der sogenannten "Diffusion Agencies» für den Diffusionsprozeß bleibt bei der Hardund Software eher unbestimmt und jedenfalls schwach. Es kann sein, daß für die Überprüfung dieser Hypothese die Kommunikationsräume mit den MS-Regionen zu eng definiert wurden.

Die Ursachen der fehlenden Adoption von Telefax in bestimmten Regionen liegen in ihrer Wirtschaftsstruktur (keine Betriebe, die die Innovation besonders wirtschaftlich einsetzen können), ihrer peripheren Lage und den distanzabhängigen Übermittlungstarifen.

Insgesamt zeigen die geschätzten Funktionen zwar echte Lageunterschiede der Gruppen an, was mit einer hohen Signifikanz belegt wird. Die angegebenen Irrtumswahrscheinlichkeiten lassen eine Interpretation der Koeffizienten der Variablen zu. Das niedrige Bestimmtheitsmaß der Schätzungen weist aber darauf hin, daß wichtige Einflußgrößen nicht in die Funktion aufgenommen wurden. Sie werden in den spezifischen Eigenheiten der einzelnen Betriebe vermutet.

Angesichts des Bestimmtheitsmaßes der Schätzungen darf der Einfluß räumlicher Faktoren nicht überbewertet werden, zumal ihre Bedeutung im Verlauf des Diffusionsprozesses abnimmt. Wie der Vergleich der komplexen Neuerung EDV und der einfacher einzusetzenden Telefax-Geräte zeigt, findet aber ein Ausgleich insbesondere bei teureren, nicht ohne weitere Anpassungen im Betrieb zu installierenden Innovationen, nicht allzu schnell statt: Räumlich unterschiedliche Diffusionsmuster sind auch wegen räumlich ungleich verteilten anderen Einflußgrößen noch lange erkennbar. 


\section{Anmerkungen}

'Für eine Erklärung und Umschreibung dieser Dienste, vgl. Schweizerische PTT-Betriebe (1982): Kommunikationsleitbild, Definitionen (Anhang 1)

${ }^{2}$ Die wichtigsten Ergebnisse dieser Umfrage sind in Buchform publiziert, vgl. Kühn R. und Müller H. (1984): EDV-Einsatz in Schweizer Betrieben 1983, Freiburg

${ }^{3}$ Die Abgrenzung der MS-Regionen (MS = Mobilité spatiale) ist ausführlich dargestellt bei Schuler M. und Nef R. (1983): Räumliche Typologien des schweizerischen Zentren-Peripherien-Musters, Arbeitsbericht des Nationalen Forschungsprogrammes "Regionalprobleme", Nr. 35, Bern

${ }^{4}$ Schweizerische PTT-Betriebe, Teilnehmerverzeichnis Telefax, Ausgabe 1980, April 1981, Juli 1982, März 1983 und März 1984

${ }^{5} \mathrm{Zu}$ den peripheren Regionen gehören die Raumtypen "agrarisch-touristische Peripherie", "agrarische Peripherie" und "agrarisch-industrielle Peripherie", vgl. Schuler und Nef 1983, S. $114 f$.

\section{Summary \\ The Diffusion of New Information Technologies in Switzerland}

This article analyses the reasons for the spatial variations in the diffusion process of innovations of the new information technology.

A model of the diffusion process is developped in which hypotheses from well-known economic and geographical research have been integrated and combined with hypotheses which have been derived from the peculiarities of the examined innovations. The model is tested with statistical methods such as discriminant analysis.

Statistical evaluation of three different innovations (electronic data processing, data processing by means of telecommunications and facsimile machines shows empirical evidence for several hypotheses though the estimated functions are only moderatly correlated with the explained variables. The most important variables of the decision to adopt the new information technology by firms are the size of plants and a high proportion of routine information activities. Spatial factors have much less influence but may prove statistically significant.

\section{Literaturverzeichnis}

APRILE, G./HOTZ-HART, B./MÜDESPACHER, A. (1984): Raumwirtschaftliche Konsequenzen neuer Kommunikationstechnologien. In: DISP (ORL) Nr. 75, S. 13-19.

BROWN, L. A. (1981): Innovation Diffusion. A New Perspective, London.

EWERS, H.J. (1984): Räumliche Innovationsdisparitäten und räumliche Diffusion neuer Technologien. In: Brugger, E.A. (Hrsg.): Regionale Innovationsprozesse und Innovationspolitik, Bern, S. 97-118.

FISCHER, G./RUTISHAUSER, P./BAUMELER, J. (1983): Räumliche Einkommensdisparitäten in der Schweiz: Das persönlich verfügbare Einkommen nach Regionen, 1970 und 1980, Arbeitsbericht des nationalen Forschungsprogramms "Regionalprobleme" No. 40, Bern.

GEILINGER, U. (1984): Ausmass, Ursachen und Folgen der funktionalen Arbeitsteilung zwischen Regionen in der schweizerischen Wirtschaft, Diss. ETH Zürich.

GRILICHES, Z. (1957): Hybrid Corn: an Exploration in the Economics of Technological Change. In: Econometrica, vol. 25, S. 501-522.

HÄGERSTRAND, T. (1953): Innovation Diffusion as a Spatial Process, Chicago/London 1967 (Schwedische Publikation 1953).

KENNEDY, C. and THIRLWALL, A.P. (1972): Surveys in Applied Economics: Technical Process. In: Economic Journal, vol. 82, S. 11-72.

LASUEN, J.R. (1973): Urbanisation and Development - the Temporal Interaction between Geographical and Sectoral Clusters. In: Urban Studies, vol. 10, S. 163-188.

MACHLUP, F. (1962): The Production and Distribution of Knowledge in the United States, Princetown.

MALECKI, E.J. (1981): Product Cycles, Innovation Cycles and Regional Economic Change. In: Technological Forecasting and Social Change, vol. 19, S. 291-306.

MANSFIELD, E. (1968): The Economics of Technological Change, New York.

MÜDESPACHER, A. (1984): Diffusionsprozesse technischer Innovationen - Erklärungsansätze für die Neuerung in der Telekommunikations- und Informationstechnologie, ETH-Forschungsprojekt MANTO, Teilbericht 2. 17.3., Zürich.

NABSETH, L. and RAY, G. F. (1972): Neue Technologien in der Industrie. Eine internationale Studie über die Verbreitung von acht Produktionsverfahren, Berlin.

OECD (1981): Information, Activities, Electronics and Telecommunications Technologies, Paris.

PRED, A. R. (1976): The Interurban Transmission of Growth in Advanced Economies: Empirical Findings versus Regional Planning Assumptions. In: Regional Studies, vol. 10, S. 151-171.

SCHMOOKLER, J. (1972): Patents, Invention and Economic Change, Cambridge, Mass.

TARDE, G. (1895): Les Lois d'Imitation, Paris. 